\title{
Anomalous diffusion at percolation threshold in high dimensions on $10^{18}$ sites
}

\author{
Dirk Osterkamp, Dietrich Stauffer and Amnon Aharony ${ }^{1}$
}

Institute for Theoretical Physics, Cologne University, D-50923 Köln, Euroland

1 visiting from School of Physics and Astronomy, Raymond and Beverly Sackler Faculty of Exact Sciences, Tel Aviv University, Ramat Aviv, Tel Aviv 69978, Israel

e-mail: osterkamp@gmx.de, stauffer@thp.uni-koeln.de, aharony@post.tau.ac.il

Using an inverse of the standard linear congruential random number generator, large randomly occupied lattices can be visited by a random walker without having to determine the occupation status of every lattice site in advance. In seven dimensions, at the percolation threshold with $L^{7}$ sites and $L \leq 420$, we confirm the expected time-dependence of the end-to-end distance (including the corrections to the asymptotic behavior).

Keywords: Monte Carlo, ant in labyrinth, random number generators, anomalous diffusion

For transport in disordered media, like the ant-in-the-labyrith model of random percolation $[1,2,3]$, one usually first constructs the disordered medium, and then starts the transport medium. In a hypercubic lattice of linear size $L$ in $d$ dimensions, one first has to decide about the status of $L^{d}$ sites. In two dimensions this is efficient since for long enough time every site is visited. In higher dimensions, however, only a small fraction of the lattice is visited and it is more efficient to determine the status of a site only when it is visited first, keeping this status fixed for later visits. We achieve this aim here for the case that every site is randomly allowed for the random walker with probability $p$ and forbidden with probability $1-p$. We apply this method to seven-dimensional percolation $[4,5]$ at its threshold $p_{c}=0.088951$ where an infinite network of allowed sites is just possible [6]. We found good agreement of the new method $(L \leq 420)$ with the traditional one $(L=23)$, while for smaller $L$ finite-size effects are seen.

Lattice sites $i=1,2, \ldots L^{d}$ are numbered in helical order such that the neighbours of site $i$ are $i \pm 1, i \pm L, i \pm L^{2}, \ldots, i \pm L^{d-1}$. In the normal technique, we fill one site after the other using consecutive random numbers $0<x_{i}<1$; if $x_{i}<p$ the site is allowed. With a simple linear congruential random number generator like

$$
j_{i+1}=k j_{i} \quad \bmod m ; \quad m=2^{n}
$$

for integers $j_{i}$ instead of real numbers $x_{i}$, on computers with at least $n$ bits per word, this method means that a step of the random walker to the right $(i \rightarrow i+1)$ corresponds to a 
multiplication of $j_{i}$ by $k$, a step to the left to a multiplication [7] by the inverse $k^{*}$ such that $k k^{*}=1$ modulo $m$. This inverse exists if and only if $k$ and $m$ are relatively prime; then $k^{*}$ can easily be calculated by the extended Euclid algorithm [8]. Tables of pairs $k, k^{*}$ were given by L'Ecuyer [9] or can be easily calculated by a Fortran program available from DO; for example $k=16807$ works with $k^{*}=1278498327$ for $n=32$ and $k^{*}=4409460005719528983$ for $n=63$. We used on a 64-bit Cray-T3E

$$
k=3512401965023503517, \quad k^{*}=3753721746144068021,
$$

table 5 of [9]. If a step upwards, $i \rightarrow i+L$, corresponds to $L$ multiplications with $k$ (modulo $m$ ), then a downwards step corresponds to $L$ multiplications with $k^{*}$ (modulo $m$ ), i.e. $L$ pseudodivisions by $k$. The other directions involve higher powers of $L$. In this way an arbitrary walk can be followed in a reproducible way by the proper number of multiplications with $k$ and $k^{*}$, always using the modulo restriction. The number of sites is then only limited by the period of the random number generator; we used a multiplier [9] with good spectral results and maximal period $2^{61}$. This method can be extended to the more general generator $j_{i+1}=k j_{i}+c \bmod m$.

We use helical boundary conditions in $d-1$ directions and unlimited extent in the remaining direction, i.e. site $i$ is thought to have as its right neighbour the site $i+1$ even if $i$ is an integer multiple of $L$ and lies at the right boundary. And if due to a large jump the new site has $i \leq 0$ or $i>L^{d}$ we do not have to put $i$ back into the traditional interval $1 \leq i \leq L^{d}$ through $i \rightarrow i \pm L^{d}$ since the new method does not actually store an array with index $i$ from 1 to $L^{d}$. In this sense our lattice size is infinite in one direction. (It is easiest to imagine a planar lattice, with sites numbered in a typewriter fashion.)

In our 32-bit Fortran program, instead of the modulo function we used the automatic omission of leading bits if a product of two integers gives an integer with more than 32 bits. Since Fortran does not have the unsigned long integer type of $\mathrm{C}$, the resulting products between $-2^{31}$ and $+2^{31}$ are often negative. We tested by comparison with the traditional method that the new technique works nevertheless. For 64 bits, we masked off the leading bit, thus working modulo $2^{63}$ with only positive integers. This program including all shmem commands for communication between different Cray processors still fits onto 68 lines, Fig. 1. It can also be used for less than seven dimension by reducing the parameter idim; we made test runs with $1,100,000,000^{2}, 1,050,000^{3}, 32800^{4}, 4100^{5}, 1001^{6}$ sites, as well as $180^{8}$.

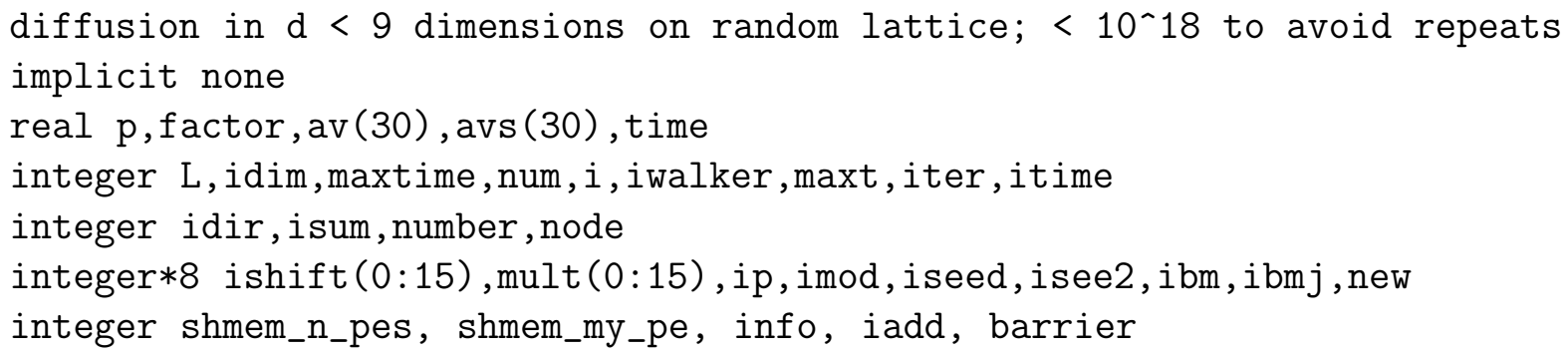




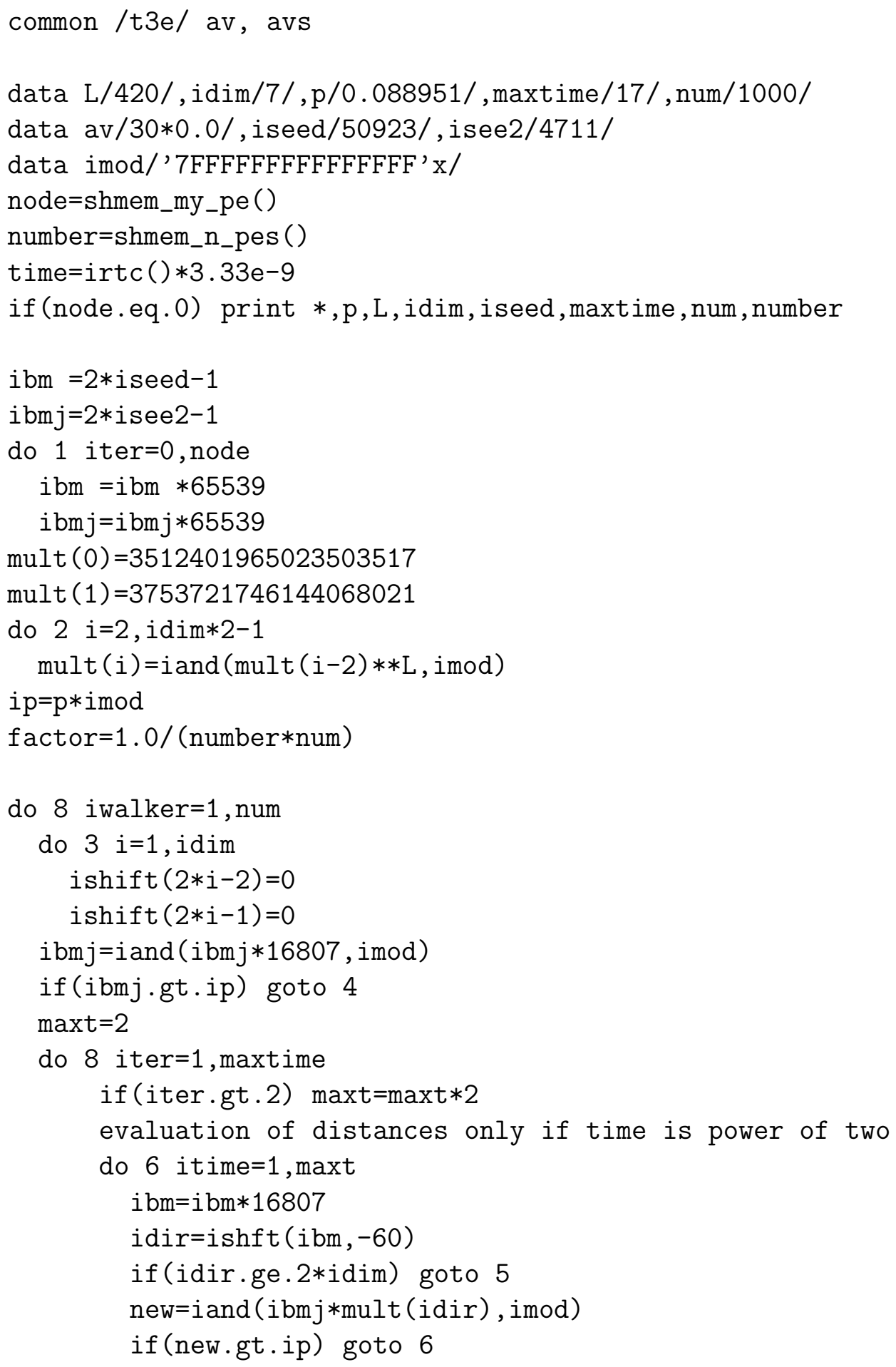




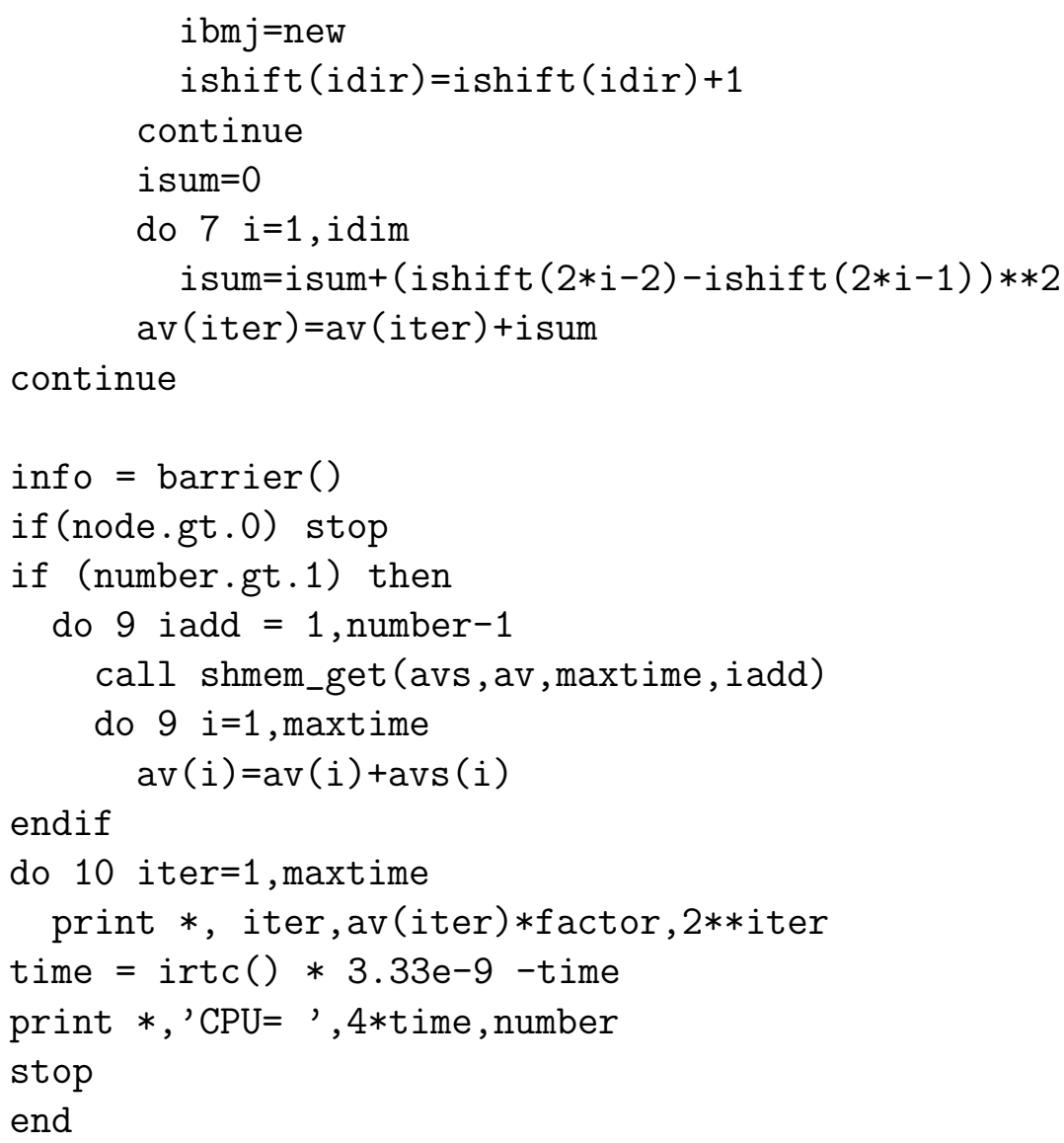

Figure 1: Fortran program for Cray-T3E parallel computing, averaging over number processors each simulating num random walkers. Without the complications of parallel computation, loops 1 and 9 as well as all commands involving shmem, barrier, node, number, avs can be omitted.

Now we repeat the standard scaling theory $[1,2,3,6]$ for the average end-to-end distance $r(t, p)$ and enlarge it by predicting correction terms.

Let $R_{s},\left\langle r_{s}(t)>,\langle r(t)>\right.$ be the average radius of gyration of a cluster containing $s$ sites, the average end-to-end distance travelled on such a cluster in time $t$, and this distance averaged over all starting points on all clusters. Analogous notations hold for the squared distances; these averages $\left\langle r^{2}(t)>\right.$ over the squares do in general not scale like the squares of 
the averages $\langle r(t)\rangle$. The number of clusters containing $s$ sites each is $n_{s}=s^{-\tau} f\left[\left(p_{c}-p\right) s^{\sigma}\right]$ with the standard critical exponents $\sigma=1 /(\beta \delta), \tau=2+1 / \delta$. In general dimensions $d$, we have $R_{s}=s^{\sigma \nu} h\left[\left(p_{c}-p\right) s^{\sigma}\right]$ with the correlation length exponent $\nu$, and assume

$$
<r_{s}^{2}(t)>=R_{s}^{2} g\left(t / s^{x}, R_{s} / s^{\sigma}\right)
$$

Right at $p=p_{c}$ this assumption simplifies to

$$
<r_{s}^{2}(t)>=R_{s}^{2} G\left(t / s^{x}\right)
$$

(varying as $R_{s}^{2}$ for $t \gg s^{x}$, when every site on the cluster has been visited many times, and as $t^{2 \sigma \nu / x}=t^{2 / d_{w}}$, similar to the anomalous behavior on the infinite cluster, for $\left.1 \ll t \ll s^{x}\right)$. For $p<p_{c}$ and for sufficiently long times one has only the former behavior: the random walker has visited the whole finite cluster, and thus $r_{s}(t=\infty) \simeq R_{s}$. Here, $f, h, g, G, g_{1}, g_{2}, \ldots$ are suitable scaling functions.

Thus below $p_{c}$ for long times we have

$$
\begin{aligned}
& <r(t)>=\sum_{s} R_{s} s n_{s} g_{1}\left[\left(p_{c}-p\right) s^{\sigma}\right] \\
& <r^{2}(t)>=\sum_{s} R_{s}^{2} s n_{s} g_{2}\left[\left(p_{c}-p\right) s^{\sigma}\right]
\end{aligned}
$$

in $d$ dimensions.

For $d>6$ the critical exponents should be those of the Bethe lattice, with $\tau=5 / 2, \sigma=$ $\nu=1 / \delta=1 / 2, n_{s} \propto s^{-5 / 2} \exp \left[-\operatorname{const}\left(p_{c}-p\right)^{2} s\right]$. Also, $d_{w}=6, x=3 / 2$. Then

$$
\begin{gathered}
<r(t)>\rightarrow \text { Const }+\ldots\left(p_{c}-p\right)^{1 / 2} \\
<r^{2}(t)>\propto \log \left(p_{c}-p\right)+\ldots
\end{gathered}
$$

for $d>6, p<p_{c}$. Right at $p=p_{c}$ the powers of $p_{c}-p$ are replaced by the proper powers of $t \sim 1 /\left(p_{c}-p\right)^{3}:$

$$
\begin{aligned}
<r(t) & >\rightarrow \text { Const }+O\left(1 / t^{1 / 6}\right) \\
& <r^{2}(t)>\propto \log t
\end{aligned}
$$

More precisely, in $\left\langle r^{2}(t)>\propto \sum_{s} s^{-1} g\left(t / s^{x}\right.\right.$, const) we can approximate the sum by $\sum_{s} s^{-1}$ with an upper limit for $s$ of order $t^{1 / x}$, giving $\ln ($ const $t)$ or Const $+\ln t$.

For the third-leading term in $\left\langle r^{2}(t)>\right.$ at $p=p_{c}$, we note that the leading correction to scaling for $d>6$ comes from the leading irrelevant parameter $w$, which represents the probability of having vertices with three bonds [10]. This implies corrections to any leading 
power law behavior of the form $\left(1+\right.$ const $\left.\times w^{2} X^{6-d}\right)$, where $X$ represents an appropriate length scale, which could be $r(t), R_{s}$ or $t^{1 / d_{w}}$. Specifically, at $p_{c}$ we expect corrections to $\left\langle r^{2}(t)>\right.$ of relative order $t^{(6-d) / 6}$, which become $1 / t^{1 / 6}$ at $d=7$, just as for $\langle r(t)>$ above. The two corrections differ for $d>7$. Thus finally at $d=7$ we expect:

$$
<r^{2}(t)>\propto \log t+\text { Const }+O\left(1 / t^{1 / 6}\right)
$$

Obviously, Eq. (1) is easier to test and reasonably confirmed by Fig. 2, using $p_{c}=0.088951$ from [5]. For Eq. (2) we see in Fig. 3 a logarithmic long-time behaviour for $L=23$ (traditional method) and $L=420$ (new method), while for short times and/or small lattices deviations exist. Figure 4, similar to Fig. 2, shows that $\left\langle r^{2}(t)\right\rangle=1.25 \ln (t)-7.8+8 / t^{1 / 6}$ is consistent with our data for large systems over 8 decades in time. $\left(<r^{2}(t=1)=p\right.$ exactly, but the curve shown in Fig. 4 extrapolates to a value at $t=1$ differing from this value by 0.2 ,) The fit is not shown in Fig. 3 since it would barely be distinguishable there from the curve for $L=420$. Less extensive simulations in eight dimensions, $L=180$, nicely confirm $\left\langle r^{2}\right\rangle \propto \log (t)+$ const, but are not accurate enough to distinguish between correction exponents 1/3 and 1/6. In contrast, for seven dimensions $1 / 6$ fits over a wider range than $1 / 3$.

In summary, the new method gives results consistent with the old one but allows for enormously larger lattice sizes; and these results are consistent with scaling theory.

We thank Humboldt Foundation and GIF for supporting this collaboration, and R.M. Ziff, P. L'Ecuyer and P.M.C. de Oliveira for encouraging discussion.

\section{References}

[1] D. Ben-Avraham and S. Havlin, J. Phs. A 15, L 691 (1982).

[2] Y. Gefen, A. Aharony and S. Alexander, Phys. Rev. Lett. 50, 77 (1983).

[3] S. Havlin and D. Ben-Avraham, Adv. Phys. 36, 695 (1987).

[4] D. Stauffer and R.M. Ziff, Int. J. Mod. Phys. C 11, 205 (2000).

[5] P. Grassberger, e-print cond-mat/0202144

[6] D.Stauffer and A.Aharony, Introduction to Percolation Theory (Taylor and Francis, London, 1994); A.Bunde and S.Havlin, Fractals and Disordered Systems, (Springer, BerlinHeidelberg 1996); M.Sahimi, Applications of Percolation Theory (Taylor and Francis, London, 1994). 
[7] G.A.Jones, Elementary Number Theory, Springer, London 1998.

[8] D.E.Knuth, The Art of Computer Programming, vol. 2 Seminumerical algorithms, 3rd ed., Addison Wesley, New York 1998.

[9] P. L'Ecuyer, Mathematics of Computation 68, 249 (1999) and erratum http://www.iro.umontreal.ca/ lecuyer/myftp/papers/latrules.err; see also H.Vollmayr, J. Stat. Phys. 74, 919 (1994) ; G. Paul, R.M. Ziff and H.E. Stanley, Phys. Rev. E 64, 026115 (2001).

[10] A. Aharony, Y. Gefen and A. Kapitulnik, J. Phys. A 17, L197 (1984).

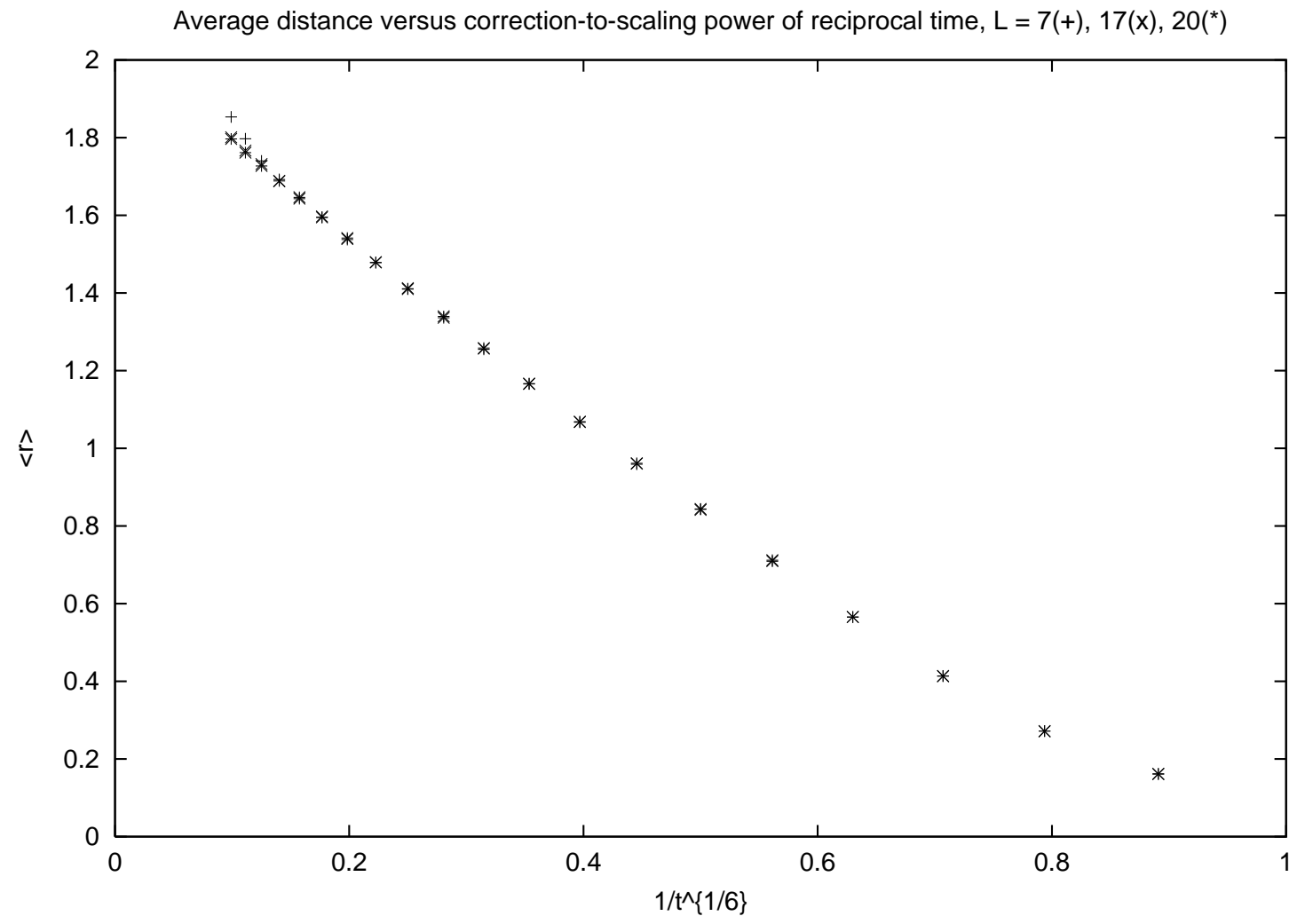

Figure 2: Average distance $\langle r\rangle$ versus $1 /$ time $^{1 / 6}$; asymptotically a straight line is expected leading to a finite intercept. 


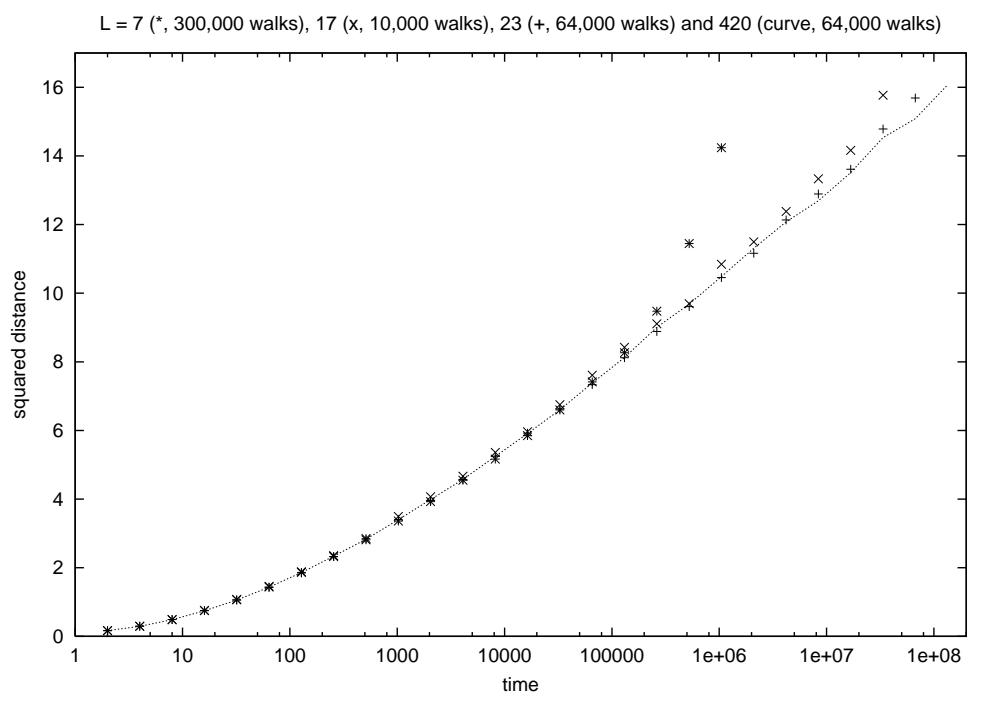

Figure 3: $\left\langle r^{2}(t)>\right.$ versus time on logarithmic time scale; $L=7$ and 17 deviate for long times from $L=23$ (traditional method) whereas $L=420$ (continuous curve, new method) agrees with $L=23$ for the observed times.

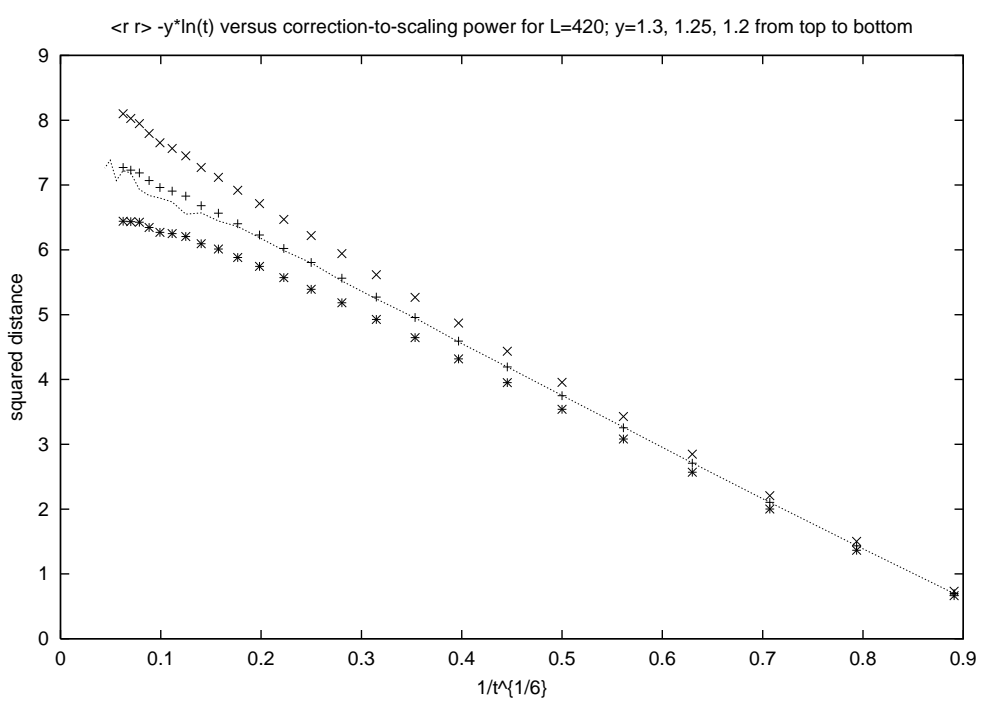

Figure 4: $\left\langle r^{2}(t)>-y \ln (t)\right.$ versus $1 /$ time $^{1 / 6}$ for $\mathrm{y}=1.2,1.25$ and $1.3 ;(640,000$ walks $)$; the line comes from 64,000 walks to eight times longer $t$ up to 128 Megasteps. Asymptotically a straight line is expected leading to a finite intercept. 